\title{
Audiological Findings in Children with Chronic Kidney Disease
}

A.A.Abdulhameed, H.R.Omar, S.M.El-Desouky and N.G.Mahmoud

Pediatrics and Otorhinolaryngology Dept., Faculty of Medicine, Benha Univ., Benha, Egypt

E-Mail: neveengamal474@yahoo.com

\begin{abstract}
Chronic renal disappointment (CRF) is characterized as the presence of kidney harm, which evaluated by estimated glomerular filtration rate (GFR). CRF has arisen as a worldwide general wellbeing trouble for its expanding number of patients, high danger of movement to end-stage renal illness (ESRD), and helpless forecast of dreariness and mortality Multiple common danger factors for ongoing kidney sickness (CKD) and hearing misfortune incorporate age, diabetes, hypertension, and prescriptions that are both ototoxic and nephrotoxic. In addition, in patients with set up CKD, different danger factors have been speculated to cause hearing misfortune including the utilization of ototoxic prescriptions, hypertension, and diabetes, especially in relationship with hypertension, electrolyte aggravations, and hemodialysis itself. The two organs are associated with body liquid homeostasis, The internal ear and the kidney share a progression of fundamental cycles for water and particle guideline just as some particular cell water channels known as aquaporins, which are known to have a vital function in the practical movement of the two organs. the two organs have epithelium containing a sodium-potassium energy-requiring measures connected to the severe need of keeping up the equilibrium of particles and a steady $\mathrm{pH}$. Point of the Work: to decide the presence, type, and seriousness of hearing misfortune (HL) in patients with constant kidney sickness on ordinary hemodialysis and on moder

ate administration and to assess the relationship of announced HL with the span of hemodialysis of kids with persistent renal disappointment on standard hemodialysis. This examination will be a case control study remembering 30 patients with ongoing renal disappointment for customary hemodialysis in the Nephrology Unit, Pediatric Department, Benha University Hospitals and 20 Patients with persistent kidney sickness on traditionalist administration and 30 sound kids as a benchmark group. All youngsters are exposed to standard and expanded unadulterated tone audiometry and tympanometry. Our examination found that 43.3 percent of CKD patients on haemodialysis had sensorineural hearing misfortune and $40 \%$ of CKD patients on traditionalist administration . SNHL was predominantly at high frequencies (47.6\%) and of gentle and moderate seriousness. Our examination demonstrated that there was measurably critical connection found among SNHL and hypertension in CKD patients yet there was no huge connection found with the span of hemodialysis or hematological boundaries and biochemical boundary aside from serum sodium level.
\end{abstract}

Keywords: Chroic kidney disease, Hearing loss, Sensorineural hearing loss.

\section{Introduction}

The cochlea and kidney have comparable physiological components, in particular the dynamic vehicle of liquid and electrolytes performed by the stria vascularis and the glomerulus, separately [1]. They may likewise have normal antigenicity [2]. In spite of the fact that the gross life structures of the kidney and cochlea contrasts extensively, there are numerous similitudes at the ultrastructural level. Both contain epithelial structures in close contact with their vascular flexibly. Storm cellar layer is found firmly juxtaposed to narrow endothelium in both Bowman's case and the proximal renal tubules of the kidney and furthermore around the vessels of the stria vascularis. Furthermore, cellar film lined intercellular directs exist in both the glomerulus and the stria vascularis [3]. Besides, the epithelial cells in both the cochlea and the kidney show highlights known to be related with dynamic vehicle of liquid and electrolytes, specifically, microvilli containing various mitochondria. The two organs are engaged with body liquid homeostasis, and in this way have epithelium containing a sodium-potassium particle siphon utilizing ATPase. Carbonic anhydrase is likewise present in both the stria vascularis and the nephron
[3]. A few factors may add to the etiopathogenetic instruments of hearing misfortune in CRF including factors identified with the seriousness and span of the illness, electrolyte unsettling influences, ototoxic medications, age, comorbid conditions, for example, diabetes mellitus and hypertension, and hemodialysis [4-6]. sensorineural hearing misfortune (SNHL) is significantly more pervasive in patients with ongoing renal disappointment (CRF) than in everybody. It goes from $28 \%$ to $77 \%[7,8]$.

\section{Aim of the work}

To determine the presence, type, and severity of hearing loss (HL) in patients with chronic kidney disease on regular hemodialysis and on conservative management and to evaluate the relationship of reported HL with the duration of hemodialysis of children with chronic renal failure on regular hemodialysis.

\section{Patients and methods}

This study will be a case control study including 30 patients with chronic renal failure on regular hemodialysis in the Nephrology Unit, Pediatric Department, Benha University Hospitals and 20 
Patients with chronic kidney disease on conservative management and 30 healthy children as a control group. All children are subjected to standard and extended pure-tone audiometry and tympanometry Patients were selected according to the certain criteria:

\subsection{Inclusion Criteria}

All patients diagnosed as chronic kidney disease including patients on regular hemodialysis and others on conservative management.

\subsection{Exclusion Criteria}

Children who had a suggestive history of congenital hearing loss, birth asphyxia, hyperbilirubinemia, or head trauma.

Children who could not cooperate during the audiometric examination will to be excluded.

\section{Results}

Our study found that 43.3 per cent of CKD patients on haemodialysis had sensorineural hearing loss and $40 \%$ of CKD patients on conservative management. SNHL was mainly at high frequencies (47.6\%) and of mild and moderate severity.

Table (1) Comparison between Control and Patients group regarding sensorineural hearing loss(SNHL) :\{type, severity and laterality and conductive hearing loss.

\begin{tabular}{|c|c|c|c|c|c|c|c|c|}
\hline & & \multicolumn{2}{|c|}{ Control } & \multicolumn{2}{|c|}{ Patients group } & \multirow{2}{*}{$\begin{array}{c}\text { Test } \\
\text { value* }\end{array}$} & \multirow{2}{*}{ P-value } & \multirow{2}{*}{ Sig. } \\
\hline & & No. & $\%$ & No. & $\%$ & & & \\
\hline \multirow{2}{*}{ SNHL } & Negative & 49 & $98.0 \%$ & 29 & $58.0 \%$ & \multirow{2}{*}{23.310} & \multirow{2}{*}{0.000} & \multirow{2}{*}{ HS } \\
\hline & Positive & 1 & $2.0 \%$ & 21 & $42.0 \%$ & & & \\
\hline \multirow{3}{*}{ Conductive HL } & Negative & 46 & $92.0 \%$ & 47 & $94.0 \%$ & \multirow{3}{*}{0.154} & \multirow{3}{*}{0.695} & \multirow{3}{*}{ NS } \\
\hline & Positive & 4 & $8.0 \%$ & 3 & $6.0 \%$ & & & \\
\hline & Low frequency & 0 & $0.0 \%$ & 3 & $14.3 \%$ & & & \\
\hline \multirow{3}{*}{ Type of SNHL } & Mid frequency & 1 & $100.0 \%$ & 8 & $38.1 \%$ & \multirow[t]{3}{*}{1.513} & \multirow[t]{3}{*}{0.469} & \multirow[t]{3}{*}{ NS } \\
\hline & High frequency & 0 & $0.0 \%$ & 10 & $47.6 \%$ & & & \\
\hline & Mild & 1 & $100.0 \%$ & 10 & $47.6 \%$ & & & \\
\hline \multirow[t]{2}{*}{ Severity of SNHL } & Moderate & 0 & $0.0 \%$ & 10 & $47.6 \%$ & \multirow[t]{2}{*}{1.048} & \multirow[t]{2}{*}{0.592} & \multirow[t]{2}{*}{ NS } \\
\hline & Severe & 0 & $0.0 \%$ & 1 & $4.8 \%$ & & & \\
\hline \multirow{2}{*}{ Which ear affected } & Unilateral & 0 & $0.0 \%$ & 1 & $4.8 \%$ & \multirow{2}{*}{0.100} & \multirow{2}{*}{0.752} & \multirow{2}{*}{ NS } \\
\hline & Bielateral & 2 & $100.0 \%$ & 20 & $95.2 \%$ & & & \\
\hline
\end{tabular}

P-value >0.05: Non significant (NS); P-value <0.05: Significant (S); P-value< 0.01: highly significant (HS) *:Chi-square test

The Previous table shows that there was highly statistically significant difference found between two groups regarding presence of SNHL and it also show the most common type of SNHL is high frequency type and most commonly of mild to moderate degrees.

While there was no statistically significant difference found between two groups regarding conductive hearing loss.

Table (2) Comparison between CKD patients on hemodialysis, CKD patients on conservative management and control group regarding serum urea, creatinine, sodium, potassium, calcium, phosphorus, hemoglobin level, total leucocytic count, platelets count, blood PH and bicarbonates level.

\begin{tabular}{|c|c|c|c|c|c|c|c|}
\hline & & $\begin{array}{c}\text { CKD on } \\
\text { hemodialysis }\end{array}$ & $\begin{array}{c}\text { CKD on } \\
\text { conservative }\end{array}$ & Control & \multirow{2}{*}{$\begin{array}{c}\text { Test } \\
\text { value• }\end{array}$} & \multirow[t]{2}{*}{ P-value } & \multirow[t]{2}{*}{ Sig. } \\
\hline & & No. $=30$ & No. $=20$ & No. $=50$ & & & \\
\hline urea & $\begin{array}{l}\text { Mean } \pm \text { SD } \\
\text { Range }\end{array}$ & $\begin{array}{c}152.13 \pm 43.23 \\
60-260\end{array}$ & $\begin{array}{c}185.30 \pm 47.70 \\
65-285\end{array}$ & $\begin{array}{c}32.70 \pm 5.87 \\
22-44\end{array}$ & 221.862 & 0.000 & HS \\
\hline create & $\begin{array}{l}\text { Mean } \pm \text { SD } \\
\text { Range }\end{array}$ & $\begin{array}{c}6.13 \pm 3.59 \\
1.8-15\end{array}$ & $\begin{array}{c}1.48 \pm 0.82 \\
0.6-3\end{array}$ & $\begin{array}{c}0.61 \pm 0.17 \\
0.3-1\end{array}$ & 74.402 & 0.000 & HS \\
\hline $\mathrm{Na}$ & $\begin{array}{c}\text { Mean } \pm \text { SD } \\
\text { Range }\end{array}$ & $\begin{array}{c}140.33 \pm 5.78 \\
133-151\end{array}$ & $\begin{array}{c}138.05 \pm 3.61 \\
134-149\end{array}$ & $\begin{array}{c}137.80 \pm 2.91 \\
130-142\end{array}$ & 3.810 & 0.026 & $S$ \\
\hline $\mathbf{k}$ & $\begin{array}{c}\text { Mean } \pm \text { SD } \\
\text { Range }\end{array}$ & $\begin{array}{c}4.62 \pm 0.85 \\
3-7\end{array}$ & $\begin{array}{c}4.28 \pm 0.69 \\
3-5.7\end{array}$ & $\begin{array}{c}3.73 \pm 0.24 \\
3-4.5\end{array}$ & 23.211 & 0.000 & HS \\
\hline ca & $\begin{array}{l}\text { Mean } \pm \text { SD } \\
\text { Range }\end{array}$ & $\begin{array}{c}4.33 \pm 0.60 \\
2.8-5.2\end{array}$ & $\begin{array}{c}4.42 \pm 0.37 \\
4-5.1\end{array}$ & $\begin{aligned} 4.47 & \pm 0.24 \\
4 & -5\end{aligned}$ & 1.145 & 0.323 & NS \\
\hline Phosphorus & $\begin{array}{c}\text { Mean } \pm \text { SD } \\
\text { Range }\end{array}$ & $\begin{array}{c}5.14 \pm 0.53 \\
4-6\end{array}$ & $\begin{array}{c}5.10 \pm 0.36 \\
4.4-5.8\end{array}$ & $\begin{aligned} 4.40 & \pm 0.34 \\
4 & -5\end{aligned}$ & 39.405 & 0.000 & HS \\
\hline $\mathbf{H b}$ & $\begin{array}{c}\text { Mean } \pm \text { SD } \\
\text { Range }\end{array}$ & $\begin{array}{c}9.26 \pm 1.50 \\
5.9-12\end{array}$ & $\begin{array}{c}10.84 \pm 1.50 \\
8-13\end{array}$ & $\begin{array}{c}10.86 \pm 0.89 \\
9.5-12\end{array}$ & 17.544 & 0.000 & HS \\
\hline Tlc & Mean \pm SD & $9.13 \pm 2.01$ & $8.93 \pm 4.31$ & $7.04 \pm 2.38$ & 6.576 & 0.002 & HS \\
\hline
\end{tabular}




\begin{tabular}{|c|c|c|c|c|c|c|c|}
\hline \multirow{2}{*}{\multicolumn{8}{|c|}{ Table (2) Continue }} \\
\hline & & & & & & & \\
\hline PIT & $\begin{array}{l}\text { Mean } \pm \text { SD } \\
\text { Range }\end{array}$ & $\begin{array}{c}241.27 \pm 72.03 \\
120-400\end{array}$ & $\begin{array}{c}301.50 \pm 108.57 \\
106-477\end{array}$ & $\begin{array}{c}258.30 \pm 66.67 \\
150-366\end{array}$ & 3.668 & 0.029 & $S$ \\
\hline PH & $\begin{array}{c}\text { Mean } \pm \text { SD } \\
\text { Range }\end{array}$ & $\begin{array}{c}7.32 \pm 0.05 \\
7.2-7.4\end{array}$ & $\begin{array}{c}7.37 \pm 0.03 \\
7.3-7.45\end{array}$ & $\begin{array}{c}7.35 \pm 0.02 \\
7.3-7.38\end{array}$ & 13.770 & 0.000 & HS \\
\hline HCO3 & $\begin{array}{c}\text { Mean } \pm \text { SD } \\
\text { Range }\end{array}$ & $\begin{array}{c}15.64 \pm 2.19 \\
13-23\end{array}$ & $\begin{array}{c}17.95 \pm 2.44 \\
13-23 \\
\end{array}$ & $\begin{array}{c}15.52 \pm 1.45 \\
13-18 \\
\end{array}$ & 12.493 & 0.000 & HS \\
\hline
\end{tabular}

P-value >0.05: Non significant (NS); P-value <0.05: Significant (S); P-value< 0.01: highly significant (HS) $\bullet$ One Way ANOVA test

The Previous table shows that there was highly statistically significant difference found between three groups regarding serum urea, creatinine, potassium, phosphorus, hemoglobin level total leucocytic count blood PH and bicarbonate level
While it was statistically significant in serum sodium and platelets count.

While there was no statistically significant difference found between three groups regarding serum calcium level.

Table (3) Comparison between CKD patients on hemodialysis, CKD patients on conservative management and control group regarding sensorineural hearing $\operatorname{loss}(\mathrm{SNHL}):\{$ type, severity and laterality $\}$ and conductive hearing loss.

\begin{tabular}{|c|c|c|c|c|c|c|c|c|c|c|}
\hline & & \multicolumn{2}{|c|}{$\begin{array}{c}\text { CKD on } \\
\text { hemodialysis }\end{array}$} & \multicolumn{2}{|c|}{$\begin{array}{c}\text { CKD on } \\
\text { conservative }\end{array}$} & \multicolumn{2}{|c|}{ Control } & \multirow[t]{2}{*}{$\begin{array}{c}\text { Test } \\
\text { value }\end{array}$} & \multirow[t]{2}{*}{$\begin{array}{c}\text { P- } \\
\text { value }\end{array}$} & \multirow[t]{2}{*}{ Sig. } \\
\hline & & No. & $\%$ & No. & $\%$ & No. & $\%$ & & & \\
\hline \multirow{2}{*}{ SNHL } & Negative & 17 & $56.7 \%$ & 12 & $60.0 \%$ & 49 & $98.0 \%$ & \multirow{2}{*}{23.388} & \multirow{2}{*}{0.000} & \multirow{2}{*}{ HS } \\
\hline & Positive & 13 & $43.3 \%$ & 8 & $40.0 \%$ & 1 & $2.0 \%$ & & & \\
\hline \multirow{2}{*}{ Conductive HL } & Negative & 30 & $100.0 \%$ & 17 & $85.0 \%$ & 46 & $92.0 \%$ & \multirow{2}{*}{4.301} & \multirow{2}{*}{0.116} & \multirow{2}{*}{ NS } \\
\hline & Positive & 0 & $0.0 \%$ & 3 & $15.0 \%$ & 4 & $8.0 \%$ & & & \\
\hline \multirow{3}{*}{ Type of SNHL } & Low frequency & 3 & $23.1 \%$ & 0 & $0.0 \%$ & 0 & $0.0 \%$ & \multirow{3}{*}{3.911} & \multirow{3}{*}{0.418} & \multirow{3}{*}{ NS } \\
\hline & Mid frequency & 4 & $30.8 \%$ & 4 & $50.0 \%$ & 1 & $100.0 \%$ & & & \\
\hline & High frequency & 6 & $46.2 \%$ & 4 & $50.0 \%$ & 0 & $0.0 \%$ & & & \\
\hline \multirow{3}{*}{$\begin{array}{l}\text { Severity of } \\
\text { SNHL }\end{array}$} & Mild & 7 & $53.8 \%$ & 3 & $37.5 \%$ & 1 & $100.0 \%$ & \multirow{3}{*}{2.587} & \multirow{3}{*}{0.629} & \multirow{3}{*}{ NS } \\
\hline & Moderate & 5 & $38.5 \%$ & 5 & $62.5 \%$ & 0 & $0.0 \%$ & & & \\
\hline & Severe & 1 & $7.7 \%$ & 0 & $0.0 \%$ & 0 & $0.0 \%$ & & & \\
\hline \multirow{2}{*}{$\begin{array}{l}\text { Which ear } \\
\text { affected }\end{array}$} & Unilateral & 1 & $7.7 \%$ & 0 & $0.0 \%$ & 0 & $0.0 \%$ & \multirow{2}{*}{0.804} & \multirow{2}{*}{0.669} & \multirow{2}{*}{ NS } \\
\hline & Bielateral & 12 & $92.3 \%$ & 8 & $100.0 \%$ & 2 & $100.0 \%$ & & & \\
\hline
\end{tabular}

The Previous table shows that there was highly statistically significant difference found between patients and control group regarding presence of SNHL but there was no statistically significant difference found between CKD patients on hemodialysis, CKD patients on conservative management.
The most common type of SNHL in CKD patients on hemodialysis was high frequency type while in CKD patients on conservative management were high and mid frequency types.

While there was no statistically significant difference found between three groups regarding conductive hearing loss and laterality of hearing loss.

Table (4) Relation between SNHL and (family history of chronic renal failure(CRF), history of ototoxic drug intake, family history of hearing loss, history of hypertension and causes of renal failure) in all cases.

\begin{tabular}{|c|c|c|c|c|c|c|c|c|}
\hline & & Nega & SNHL & Posit & SNHL & Test & P- & \\
\hline & & No. & $\%$ & No. & $\%$ & value* & value & Sig. \\
\hline F.H of CRF & No & 24 & $82.8 \%$ & 18 & $85.7 \%$ & 0070 & 0778 & NS \\
\hline & Yes & 5 & $17.2 \%$ & 3 & $14.3 \%$ & & & Niv \\
\hline History of ototoxic drugs & Negative & 29 & $100.0 \%$ & 21 & $100.0 \%$ & NA & NA & NA \\
\hline
\end{tabular}




\begin{tabular}{lcccccccc} 
F. H of Hearing loss & No & 29 & $100.0 \%$ & 21 & $100.0 \%$ & NA & NA & NA \\
\hline Table (4) Continue & & & & & & & \\
\hline History of HTN & No & 26 & $89.7 \%$ & 13 & $61.9 \%$ & 5.466 & 0.019 & S \\
& Yes & 3 & $10.3 \%$ & 8 & $38.1 \%$ & & & \\
& Nephrophthesis & 4 & $13.8 \%$ & 6 & $28.6 \%$ & & \\
& Obstructive uropathy & 10 & $34.5 \%$ & 7 & $33.3 \%$ & & \\
Cause of RF & GN & 3 & $10.3 \%$ & 1 & $4.8 \%$ & & \\
& PUV & 2 & $6.9 \%$ & 3 & $14.3 \%$ & & \\
& SLE & 3 & $10.3 \%$ & 0 & $0.0 \%$ & 7.577 & 0.577 & NS \\
& Hydrouretronephrosis & 2 & $6.9 \%$ & 1 & $4.8 \%$ & & & \\
& Congenital (single kidney) & 3 & $10.3 \%$ & 2 & $9.5 \%$ & & & \\
& HUS & 1 & $3.4 \%$ & 0 & $0.0 \%$ & & & \\
\hline
\end{tabular}

P-value >0.05: Non significant (NS); P-value <0.05: Significant (S); P-value< 0.01: highly significant (HS) $*$ :Chi-square test

The Previous table shows that there was statistically significant relation found between SNHL and hypertension.

While there was no statistically significant difference found between SNHL and family history of chronic renal failure(CRF), history of ototoxic drug intake, family history of hearing loss, and causes of renal failure

Table (5) Relation between SNHL and (serum urea, creatinine, sodium, potassium, calcium, phosphorus, hemoglobin level, total leucocytic count, platelets count, blood PH and bicarbonates level) in all cases.

\begin{tabular}{|c|c|c|c|c|c|c|}
\hline & & $\begin{array}{c}\text { Negative SNHL } \\
\text { No. }=29\end{array}$ & $\begin{array}{c}\text { Positive SNHL } \\
\text { No. }=21\end{array}$ & $\begin{array}{c}\text { Test } \\
\text { value• }\end{array}$ & $\begin{array}{c}\text { P- } \\
\text { value }\end{array}$ & Sig. \\
\hline Urea & $\begin{array}{l}\text { Mean } \pm \text { SD } \\
\text { Range }\end{array}$ & $\begin{array}{c}158.72 \pm 51.45 \\
60-285\end{array}$ & $\begin{array}{c}174.62 \pm 40.89 \\
106-260\end{array}$ & -1.172 & 0.247 & NS \\
\hline Create & $\begin{array}{c}\text { Mean } \pm \text { SD } \\
\text { Range }\end{array}$ & $\begin{array}{c}4.13 \pm 3.60 \\
0.6-15\end{array}$ & $\begin{array}{c}4.45 \pm 3.75 \\
0.6-13.6\end{array}$ & -0.298 & 0.767 & NS \\
\hline $\mathbf{N a}$ & $\begin{array}{c}\text { Mean } \pm \text { SD } \\
\text { Range }\end{array}$ & $\begin{array}{c}137.97 \pm 3.45 \\
133-146\end{array}$ & $\begin{array}{c}141.43 \pm 6.32 \\
134-151\end{array}$ & -2.489 & 0.016 & S \\
\hline $\mathbf{K}$ & $\begin{array}{c}\text { Mean } \pm \text { SD } \\
\text { Range }\end{array}$ & $\begin{array}{c}4.53 \pm 0.84 \\
3-7\end{array}$ & $\begin{array}{c}4.42 \pm 0.75 \\
3-5.7\end{array}$ & 0.464 & 0.645 & NS \\
\hline $\mathbf{C a}$ & $\begin{array}{c}\text { Mean } \pm \text { SD } \\
\text { Range }\end{array}$ & $\begin{array}{c}4.32 \pm 0.54 \\
2.8-5.2\end{array}$ & $\begin{array}{c}4.43 \pm 0.49 \\
3.1-5.2\end{array}$ & -0.744 & 0.460 & NS \\
\hline Phosphorus & $\begin{array}{c}\text { Mean } \pm \mathrm{SD} \\
\text { Range }\end{array}$ & $\begin{array}{c}5.07 \pm 0.41 \\
4.4-5.7\end{array}$ & $\begin{array}{c}5.20 \pm 0.55 \\
4-6\end{array}$ & -0.914 & 0.366 & NS \\
\hline $\mathbf{H b}$ & $\begin{array}{c}\text { Mean } \pm \mathrm{SD} \\
\text { Range }\end{array}$ & $\begin{array}{c}9.77 \pm 1.68 \\
5.9-13\end{array}$ & $\begin{array}{c}10.06 \pm 1.70 \\
7.1-12\end{array}$ & -0.599 & 0.552 & NS \\
\hline Tlc & $\begin{array}{c}\text { Mean } \pm \text { SD } \\
\text { Range }\end{array}$ & $\begin{array}{c}9.39 \pm 3.74 \\
5-24\end{array}$ & $\begin{array}{c}8.57 \pm 1.89 \\
5-11\end{array}$ & 0.924 & 0.360 & NS \\
\hline Plt & $\begin{array}{c}\text { Mean } \pm \text { SD } \\
\text { Range }\end{array}$ & $\begin{array}{c}268.76 \pm 83.83 \\
106-477\end{array}$ & $\begin{array}{c}260.67 \pm 104.97 \\
120-432\end{array}$ & 0.303 & 0.763 & NS \\
\hline $\mathbf{P h}$ & $\begin{array}{c}\text { Mean } \pm \mathrm{SD} \\
\text { Range }\end{array}$ & $\begin{array}{l}7.34 \pm 0.04 \\
7.22-7.43\end{array}$ & $\begin{array}{c}7.33 \pm 0.06 \\
7.2-7.45\end{array}$ & 0.665 & 0.509 & NS \\
\hline Hco3 & $\begin{array}{c}\text { Mean } \pm \mathrm{SD} \\
\text { Range }\end{array}$ & $\begin{array}{c}16.76 \pm 2.40 \\
13-23\end{array}$ & $\begin{array}{c}16.29 \pm 2.76 \\
13-23\end{array}$ & 0.650 & 0.519 & NS \\
\hline Conductive HL & $\begin{array}{l}\text { Negative } \\
\text { Positive }\end{array}$ & $\begin{array}{c}26(89.7 \%) \\
3(10.3 \%)\end{array}$ & $\begin{array}{c}21(100.0 \%) \\
0(0.0 \%)\end{array}$ & 2.311 & 0.128 & NS \\
\hline
\end{tabular}

P-value >0.05: Non significant (NS); P-value <0.05: Significant (S); P-value< 0.01: highly significant (HS) $\bullet$ Independent t-test

The Previous table shows that there was statistically significant relation found between SNHL and serum sodium in all cases.
The Previous table shows that there was no statistically significant difference found between SNHL and serum urea, creatinine, potassium, 
calcium, phosphorus, hemoglobin level, total leucocytic count, platelets count, blood $\mathrm{PH}$ and bicarbonates level in all cases.

\section{Discussion}

The cochlea and kidney have comparative physiological systems, to be specific the dynamic vehicle of liquid and electrolytes performed by the stria vascularis and the glomerulus, individually [1]

Alport revealed an exemplary hereditary condition with hearing shortfall and renal disappointment that was named after him. From that point forward, there has been a consistently developing interest in the consultation capacity of patients with kidney infection [9].

The frequency of end-stage renal illness (ESRD) in youngsters is significantly lower than that among grown-ups. Youngsters represent just a little portion of the all out dialysis understanding populace due to both the generally low occurrence of ESRD in kids and the broad utilization of renal transplantation among pediatric ESRD patients [10].

Around one-portion of pediatric ESRD patients have an innate or a genetic issue and one-half have an obtained renal sore [11].

Our investigation found that 43.3 percent of CKD patients on haemodialysis had sensorineural hearing misfortune,

As announced by Esfahani et al who discovered $30 \%$ had SNHL among their CRF patients [12].

As opposed to contemplates that announced by Ho $\mathrm{r}$ and Charachon who revealed higher prevelance of SNHL among patients with persistent renal disappointment $[13,14]$.

Hearing edges were strange in CKD patients on haemodialysis over all frequencies going from $250 \mathrm{~Hz}$ to $8 \mathrm{kHz}$.In our examination, most of these patients experienced high recurrence type in $46.2 \%$

As revealed by Gatland et al. who discovered 53 percent in high recurrence range [15].

As respects seriousness we found that the most well-known sort was gentle to direct level of hearing misfortune in $92.3 \%$ of patients. though extreme SNHL was discovered distinctly in $7.7 \%$ of patients and no significant SNHL was accounted for. This outcome is predictable with that of Gatland et al. [Gatland et al.,1991] however not with Esfahani et al. , who found that extreme degree was the principle finding in the 30 patients examined [12].

In our investigation, SNHL was respectively detailed in completely influenced cases. This outcome is similar to the examination by Esfahani et al. [Esfahani et al., 2004]; nonetheless, in the investigation by Nikolopoulos et al, half of patients had one-sided HL [16].

We found no critical connection of SNHL with the term of hemodialysis, which is like the consequence of Bazzi et al. [8].
Conversely, Peyvandi et al. proposed in an examination that predominance and seriousness of hearing misfortune increments with term of CKD and haemodialysis [17].

It appeared to be that standard dialysis doesn't forestall the diligence of mellow indications of uremic encephalopathy and fringe neuropathy, yet these signs don't seem to exacerbate with expanding dialytic age; In our examination there was no factually critical distinction between CKD patients on hemodialysis $(10 \%)$ and CKD patients on traditionalist administration (10\%) with respect to fringe neuropathy which is upheld by the aftereffect of Sobh et al. [18], who found no measurable contrasts between SNHL in CRF treated minimalistically and those treated by dialysis

In our investigation we found that hypernatremia was statistaically huge with hearing misfortune. Anyway serum potassium didn't show any critical relationship with hearing impairement.

Different variables may influence hearing in uremic patients are hypertension, Vitamin D inadequacy, and raised serum urea levels, are proposed systems for hearing disability in patients with CRF [8,16,19].

Our investigation demonstrated that there was factually huge connection found among SNHL and hypertension in CKD patients

Determination A high occurrence of HL among kids with CRF on standard hemodialysis and on moderate administration was found in this investigation. There was measurably critical connection found among SNHL and hypertension in CKD patients however there was no huge connection found with the term of hemodialysis or hematological boundaries and biochemical boundary aside from serum sodium level.

\section{References}

[1] O.Ozturan, S.Lam. The effect of hemodialysis on hearing using pure-tone audiometry and distortion-product otoacoustic emissions. ORL J Otorhinolaryngol Relat Spec, Vol.60, PP.306313,1998.

[2] W.Arnold, H.Weidauer. Experimental studies on the pathogenesis of inner ear disturbance in renal diseases. Arch Otorhinolaryngol, Vol.211, PP. 217,1975.

[3] C.A.Quick, A.Fish, C.Brown. The relationship between cochlea and kidney. Laryngoscope, Vol.83, PP.1469-1482,1973.

[4] P.Stavroulaki, T.P.Nikolopoulos, I.Psarommatis. Hearing evaluation with distortion-product otoacoustic emissions in young patients undergoing haemodialysis. Clin Otolaryngol Allied Sci, Vol.26, PP.23542,2001,

[5] M.K.Agarwal. A study of otorhinolaryngological manifestations in patients of chronic renal failure. Indian $\mathbf{J}$ 
Otolaryngol Head Neck Surg, Vol.49, PP.31620,1997.

[6] C.Thodi, E.Thodis, V.Danielides. Hearing in renal failure. Nephrol Dial Transplant, Vol.21,ppp.3023-30,2006.

[7] R.S.Meena, Y.Aseri, B.K.Singh. Hearing loss in patients of chronic renal failure: A study of 100 cases. Indian J Otolaryngol Head Neck Surg, Vol.64, PP.356-9,2012.

[8] C.Bazzi, C.T.Venturini, C.Pagani, . Hearing loss in short- and long-term haemodialysed patients. Nephrol Dial Transplant, Vol.10, PP.18658,1995 .

[9] A.C. Alport. Hereditary familial congenital hemorrhagic nephritis. Br Med J, Vol. 1, PP.504-506,1927.

[10] M.R.Lilien, J.W. Groothoff. Cardiovascular disease in children with CKD or ESRD. Nat Rev Nephrol, Vol. 5, PP.229-235,2009.

[11] B.A.Warady, V.Chadha. Chronic kidney disease in children: the global perspective. Pediatr Nephrol, Vol. 22, PP.1999,2009.

[12] S.T. Esfahani, A. Madani, N. Ataei, .Sensorineural hearing loss in children with endstage renal disease. Acta Med Iran, Vol.42, PP.375-378,2004.
[13] G.K. Ho“r, G. Bei. Z. Nephritis. Hais Nasen Ohrenheilkd, Vol. 8, PP.375-376,1924.

[14] R.Charachon, V.Moreno Ribes, D.Cordonnier. Deafness due to renal failure. Clinopathological study. Ann Otolaryngol Chir Cervicofac, Vol.95, PP.179-203,1978.

[15] D.Gatland, B.Tucker, S.Chalstrey. Hearing loss in chronic renal failure - hearing threshold changes following haemodialysis. J R Soc Med.vol 84, PP.587-589, 1991.

[16] T.P.Nikolopoulos, D.C. Kandiloros, J.V.Segas. Auditory function in young patients with chronic renal failure. Clin Otolaryngol Allied Sci, Vol. 22, PP.222-225,1997,

[17] A.Peyvandi, N.A.Roozbahany. Hearing loss in chronic renal failure patient undergoing hemodialysis. Indian J Otolaryngol Head Neck Surg Dec, Vol.65(Suppl 3), PP.537-40,2013.

[18] M.A.Sobh, M.M.H. El Koussi, M.S.Bakr. Value of otoacaustic emission in monitoring hearing acuity in chronic renal failure patients. Saudi J Kidney Dis Transplant, Vol.10, PP.144147,1999 .

[19] G.B. Brookes. D.Vitamin. deficiency and deafness: update. Am J Otol, Vol.6, PP.1027,1984 . 